\title{
Whole-exome sequencing identifies a novel homozygous frameshift mutation in the PROM1 gene as a causative mutation in two patients with sporadic retinitis pigmentosa
}

\author{
SANMEI LIU ${ }^{5^{*}}$, LAN XIE $^{3,4^{*}}$, JUN YUE $^{3,4}$, TAO MA $^{3,4}$, CHUNYAN PENG $^{1,2}$, \\ BIYUAN QIU ${ }^{1}$, ZHENGLIN YANG ${ }^{1,2}$ and JIYUN YANG ${ }^{1,2,4}$
}

\begin{abstract}
${ }^{1}$ Sichuan Provincial Key Laboratory for Human Disease Gene Study, Hospital of the University of Electronic Science and Technology of China and Sichuan Provincial People's Hospital; ${ }^{2}$ School of Medicine, University of Electronic Science and Technology of China; ${ }^{3}$ Department of Gynecology and Obstetrics, ${ }^{4}$ Prenatal Diagnosis Center, ${ }^{5}$ Department of Ophthalmology, Hospital of The University of Electronic Science and Technology of China and Sichuan Provincial People's Hospital, Chengdu, Sichuan, P.R. China
\end{abstract}

Received November 17, 2015; Accepted March 30, 2016

DOI: $10.3892 /$ ijmm.2016.2551

\begin{abstract}
Retinitis pigmentosa (RP) refers to a heterogeneous group of inherited retinal diseases caused by the loss of photoreceptors. The present study aimed to identify the gene mutations responsible for RP in two patients diagnosed with sporadic RP using next-generation sequencing technology. For this purpose, two patients with sporadic RP and family members (namely parents and siblings) were recruited into this study and underwent a complete ophthalmological assessment. Whole-exome sequencing (WES) was performed on genomic DNA samples isolated from peripheral leukocytes which had been obtained from the two patients diagnosed with sporadic RP. WES data were annotated and filtered against four public databases and one in-house database. Subsequently, Sanger sequencing was performed in order to determine whether any of the candidate variants co-segregated with the disease phenotype in the families. A homozygous frameshift mutation, c.1445dupT (p.F482fs) in exon 12 of the PROM1 gene (MIM: 604365), satisfied a recessive inheritance model and showed complete co-segregation of the mutation with the disease phenotype in the families. The same mutation was not detected in the 200 ethnically-matched control samples by Sanger sequencing. The novel homozygous mutation c.1445dupT (p.F482fs) in the PROM1 gene was identified as a causative mutation for RP. Thus, the identification of this mutation has further expanded the existing spectrum of
\end{abstract}

Correspondence to: Dr Jiyun Yang, Sichuan Provincial Key Laboratory for Human Disease Gene Study, Hospital of the University of Electronic Science and Technology of China and Sichuan Provincial People's Hospital, 32, Section 2, The Western First Round Road, Chengdu, Sichuan 610072, P.R. China

E-mail: yangjiyun@yeah.net

*Contributed equally

Key words: retinitis pigmentosa, whole-exome sequencing, mutation, prominin 1
PROM1 mutations in patients with RP, thereby assisting in the molecular diagnosis of RP and enhancing our understanding of genotype-phenotype correlations in order to provide effective genetic counseling.

\section{Introduction}

Retinitis pigmentosa (RP; MIM: \#268000) refers to a heterogeneous group of inherited retinal diseases (IRDs) caused by the loss of photoreceptors, which is characterized by night blindness, progressive loss of peripheral vision in the early stages, and complete loss of vision in the end stages. The worldwide prevalence of RP is reported as approximately one case in 3,500-5,000 individuals (1). RP is classified as non-syndromic and syndromic RP. Non-syndromic RP is inherited in different patterns. Approximately $30-40 \%$ of cases are autosomal dominant, 5-15\% are inherited through X-linkage and 50-60\% of cases are most likely autosomal recessive (2). Rarer forms also exist, such as mitochondrial and digenic RP. Digenic RP occurs in individuals who are heterozygous for both a retinal outer segment membrane protein 1 (ROMI) mutation and a peripherin 2 (retinal degeneration, slow) (PRPH2) mutation (3).

$\mathrm{RP}$ is extremely heterogeneous. To date, mutations in 77 genes have been found to cause non-syndromic RP [Retinal Information Network (RetNet) database, https://sph.uth.edu/ retnet/sum-dis.htm]. There may be many different diseasecausing mutations in each gene. Different mutations in the same gene may cause different diseases and the same mutation in different individuals may produce different clinical consequences, even among cases within the same family. Moreover, the spectrum of mutations within a given gene may vary between populations $(1,3,4)$. The extent of heterogeneity among patients with RP is a cause of confusion to both patients and clinicians, and is a confounding factor in the diagnosis of RP. However, the known causal genes explain no more than half of the clinical cases of RP. Novel causative genes remain to be discovered (2). The identification of the genetic mutations causing RP improves our understanding of the disease process and promotes the 
development of novel treatments. Several methods are used to detect genetic variations and of these, Sanger sequencing remains the gold-standard. Due to the heterogeneity of RP, it is time consuming and expensive to screen all known genes. Novel and efficient methods of screening mutations are therefore necessary to aid in the detection of variations in known causal genes and in the discovery of the remaining fraction of RP genes. The development of high-throughput sequencing techniques over the past 10 years has increased the power to detect variations (5). Next-generation sequencing, in particular whole-exome sequencing (WES), enables investigators to perform the analysis of the coding regions of the human genome in individuals or in small families, including patients in whom a clear genotype-phenotype correlation is absent as well as to analyze clinically and genetically heterogeneous conditions (5). Previous research has demonstrated that WES provides a promising alternative method for the molecular diagnosis and identification of disease genes in Mendelian disorders (6-9).

In the present study, we successfully used the WES approach to determine the genetic basis of sporadic RP in two Chinese patients. We have identified a causative mutation in the prominin 1 (PROM1) gene that has not been previously detected, to the best of our knowledge.

\section{Subjects and methods}

Subjects and clinical assessment. Two patients with sporadic RP (case\#1 and case\#2) as well as family members (namely parents and siblings) from China were recruited into this study. The present study was performed in accordance with the Code of Ethics of the World Medical Association (Declaration of Helsinki) for medical research involving human subjects. The Institutional Review Boards of the Hospital of the University of Electronic Science and Technology of China and Sichuan Provincial People's Hospital (Chengdu, China) approved this study. Written informed consent was obtained from all participants or their guardians. All subjects were evaluated by a retina specialist. A complete ophthalmological assessment of each family member was performed, including best-corrected visual acuity (BCVA), slit-lamp biomicroscopy, fundus photography, visual field tests (Octopus; Interzeag, Schlieren, Switzerland), and electroretinography (ERG). A clinical diagnosis of RP was based on the presence of night blindness, severe defects of the peripheral visual field, lesions in the fundus (bone-spiculeshaped pigment deposits, vessel attenuation and various degrees of retinal atrophy) and abnormal ERG measurements (marked diminution in the amplitude of a- and b-waves or the complete absence of a response) as well as family history.

The control subjects were recruited from the Hospital of the University of Electronic Science and Technology of China and Sichuan Provincial People's Hospital. All subjects provided informed consent prior to participating in the study. The 200 normal matched controls underwent an eye examination and no signs of eye diseases were observed.

DNA extraction. Blood $(5 \mathrm{ml})$ from the probands, their family members and the controls was collected in ethylenediaminetetraacetic acid (EDTA) Vacutainer tubes. All genomic DNA was isolated from the peripheral leukocytes using a QIAamp DNA Blood Midi kit (Qiagen, Hilden, Germany) according to the manufacturer's instructions. The DNA samples were stored at $-20^{\circ} \mathrm{C}$ until use. DNA integrity was evaluated by performing $1 \%$ agarose gel electrophoresis.

WES and data analysis. WES was performed on the genomic DNA samples obtained from the two patients with sporadic RP by Axeq Technologies, Inc. (Seoul, Korea). The samples for sequencing were prepared according to the Illumina protocols. Illumina Exome Enrichment and quality control analysis for the enriched library were performed by Axeq Technologies Inc. A TruSeq Exome Enrichment kit was used to enrich the coding regions of the human genome. It covered 20,794 genes and 201,121 exons in the Consensus Coding Sequence (CCDS) Region database, and approximately $97.2 \%$ of CCDS exons or $96.4 \%$ of Reference Sequence (RefSeq) exons were captured (http://www.illumina.com/products/ truseq-exome.html).

WES data analysis was performed as described previously $(10,11)$. Briefly, raw data were processed by Illumina base-calling software 1.7 using default parameters. The sequencing reads were aligned to the human reference genome [hg19; University of California, Santa Cruz (UCSC) Genome Browser; http://genome.ucsc.edu/] using Burrows-Wheeler Aligner (BWA) software (http://bio-bwa.sourceforge.net/). Sequence variants [single nucleotide polymorphisms (SNPs) and short insertions or deletions (indels)] were listed. The variants were annotated and filtered against the following four public databases and one in-house database: dbSNP138 (http://www.ncbi.nlm.nih.gov/projects/SNP/), 1000 Genomes Project (ftp://ftp.1000genomes.ebi.ac.uk/vol1/ftp), HapMap Project (ftp://ftp.ncbi.nlm.nih.gov/hapmap), Exome Variant Server for the NHLBI Exome Sequencing Project (http://evs.gs.washington.edu/EVS/), Exome Aggregation Consortium (ExAC) database (http://exac.broadinstitute.org/) and our in-house database generated from 1,977 WES samples. Two major steps were performed to prioritize all the variants: i) synonymous variants, intronic variants ( $>5 \mathrm{bp}$ from exon boundaries) and common variants (minor allele frequency, $>1 \%$ ) were excluded from downstream analysis; ii) possible deleterious effects of each variant on protein structure/function were predicted using the SIFT algorithm (http://sift.bii.a-star. edu.sg/) and Polymorphism Phenotyping v2 (Polyphen2; http:/genetics.bwh.harvard.edu/pph2/). The variants were classified as potentially pathogenic variants, variants of unknown clinical significance or benign variants, in accordance with the interpretation guidelines of the American College of Medical Genetics and Genomics (ACMG) (12). Deleterious mutations and variants of unknown clinical significance were further classified as being related or unrelated to the proband phenotype. In the present study, we focused on only 238 disease-causing genes for IRDs, and prioritized 77 disease-causing genes for non-syndromic RP using the RetNet database (https://sph.uth. edu/retnet/sum-dis.htm).

Validation of variants. After filtering the variants against multiple databases, Sanger sequencing was used to determine whether any of the candidate variants co-segregated with the disease phenotype in these families. The primers were designed according to the genomic sequences of the Human Genome database and synthesized by Sangon Biotech (Shanghai, China). 

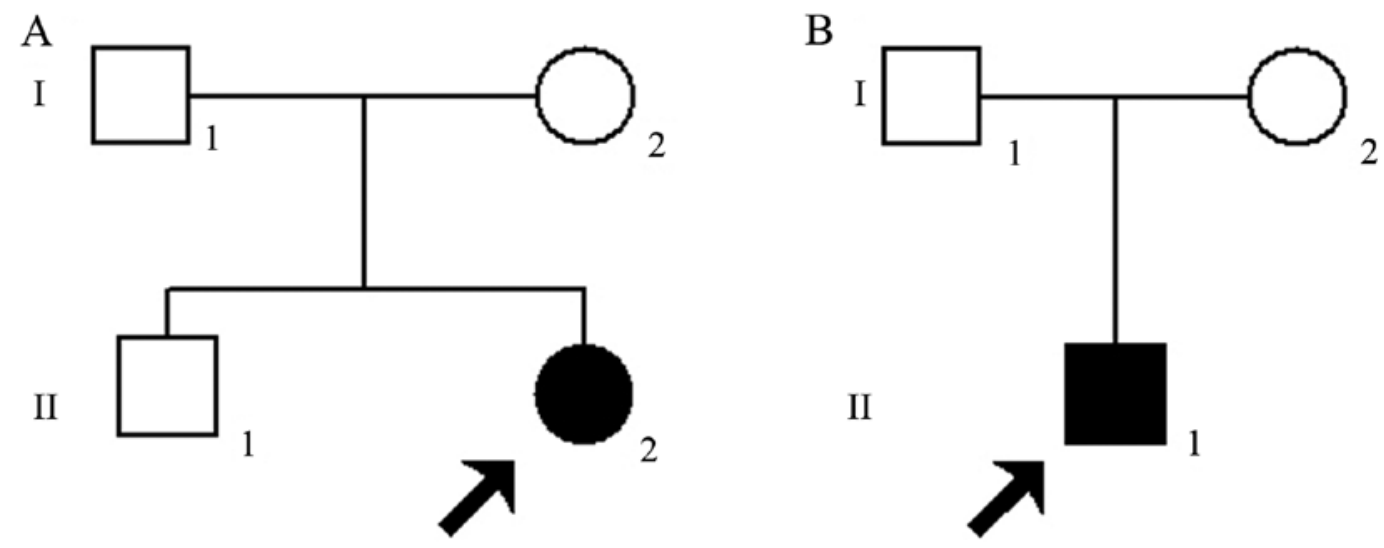

Figure 1. Pedigree of family with retinitis pigmentosa (RP). (A) Pedigree of case\#1 with RP. (B) Pedigree of case\#2 with RP. Solid symbols indicate affected individuals. Open symbols indicate unaffected individuals and black arrows indicates the probands.

Sanger sequencing was performed according to the manufacturer's protocols and the processed samples were analyzed on an ABI 3730XL Genetic Analyzer (Applied Biosystems Life Technologies, Foster City, CA, USA).

\section{Results}

Clinical data of the patients. Case\#1 (Fig. 1A) developed night blindness at the age of 8 . She presented with severely impaired visual acuity [oculus dexter (OD), right eye, 0.05 ; oculus sinister (OS), left eye: 0.05$]$ and severe defects of the peripheral visual field at 26 years of age. Case\#2 (Fig. 1B) experienced defective dark adaptation at 14 years of age. At the age of 23 , the patient presented with markedly decreased visual acuity (OD, 0.04; OS, 0.04 ) in both eyes, and severe defects of the peripheral visual field.

Fundus examination of the probands showed bonespicule-shaped pigment deposits, retinal vessel attenuation as well as retinal atrophy (Fig. 2A and B). Electroretinograms revealed no recordable responses under either scotopic or photopic conditions, indicating a significant loss of function of both the rods and the cones (Fig. 2C). An ophthalmological examination of the other family members confirmed that they did not exhibit symptoms of RP (data not shown). Since the parents of the probands had no apparent symptoms of RP, the disease shows a pattern of recessive inheritance in these families. In addition, regarding de novo mutation, it is still possible that the disease shows a pattern of autosomal dominant inheritance in these families.

Identification of disease-causing candidate variants. To identify the causative mutation in the patients affected by RP, WES was performed on the genomic DNA with mean read depths across the targeted regions (42.3x and 57.6x) for each proband. In the genomic sample from case\#1, 69,584 variants were initially identified, including 18,753 variants in the exonic and splicing regions. WES data analysis of the sample from case \#2 identified 72,048 variants, including 20,690 variants in the exonic and splicing regions. We prioritized the functional SNPs/indels in homozygous or heterozygous mutations, including non-synonymous (NS) variants, splice acceptor and donor site mutations (SS), and frameshift indels in coding regions, which were more likely to be pathogenic mutations. These variants were then compared with the databases (dbSNP138, 1000 Genomes Project, HapMap Project, ExAC database and our in-house database generated from 1,977 WES samples). We focused on only 238 diseasecausing genes for IRDs and prioritized 77 disease-causing genes for non-syndromic RP. Homozygous or compound heterozygous variants were filtered for potential autosomal recessive RP-causing mutations. Regarding de novo mutations, it is still possible that the disease shows a pattern of autosomal dominant inheritance in these families. Heterozygous variants in the disease-causing genes for autosomal dominant retinal diseases were also filtered for potential causative mutations. The filtered data were narrowed down to 4 variants in case\#1 and 10 variants in case\#2 (Table I).

Mutation detection and validation by Sanger sequencing. Three variants in case\#1 (DH3B, ABCA4 and TULP1) were excluded as autosomal recessive-IRD-causing mutations. A homozygous frameshift mutation, c.1445dupT (p.F482fs) in exon 12 of PROM1 (MIM: 604365) satisfied a recessive inheritance model. Sanger sequencing confirmed that the affected sibling (case\#1 proband, II: 2) was homozygous for c.1445dupT (p.F482fs) whereas her parents (I: 1 and I:2) and older brother (II: 1) were unaffected heterozygous carriers of c.1445dupT (p.F482fs), showing complete co-segregation of the mutation with the disease phenotype in this family (Fig. 3). Five candidate variants for autosomal dominant-IRD-causing mutations (HARS, NR2E3, OPAl, $S E M A 4 A$ and WFS1) were found in the samples from the father or the mother of case $\# 2$ by Sanger sequencing. The c.1445dupT mutation was also observed in case\#2 (Fig. 3). Furthermore, the parents of case\#2 were found to carry the same heterozygous mutation.

This mutation was not detected by Sanger sequencing in 200 ethnically-matched control samples (data not shown). Taken together with the clinical presentation of the patients with RP, these data demonstrate that the homozygous mutation, c.1445dupT (p.F482fs) in the PROMI gene is a causative mutation for RP. The frame-shift mutation disrupts the amino acid sequence starting at position 482 ; this region is highly evolutionarily conserved for $P R O M 1$, which was confirmed by the analysis of orthologs from 8 different species (Fig. 4). 

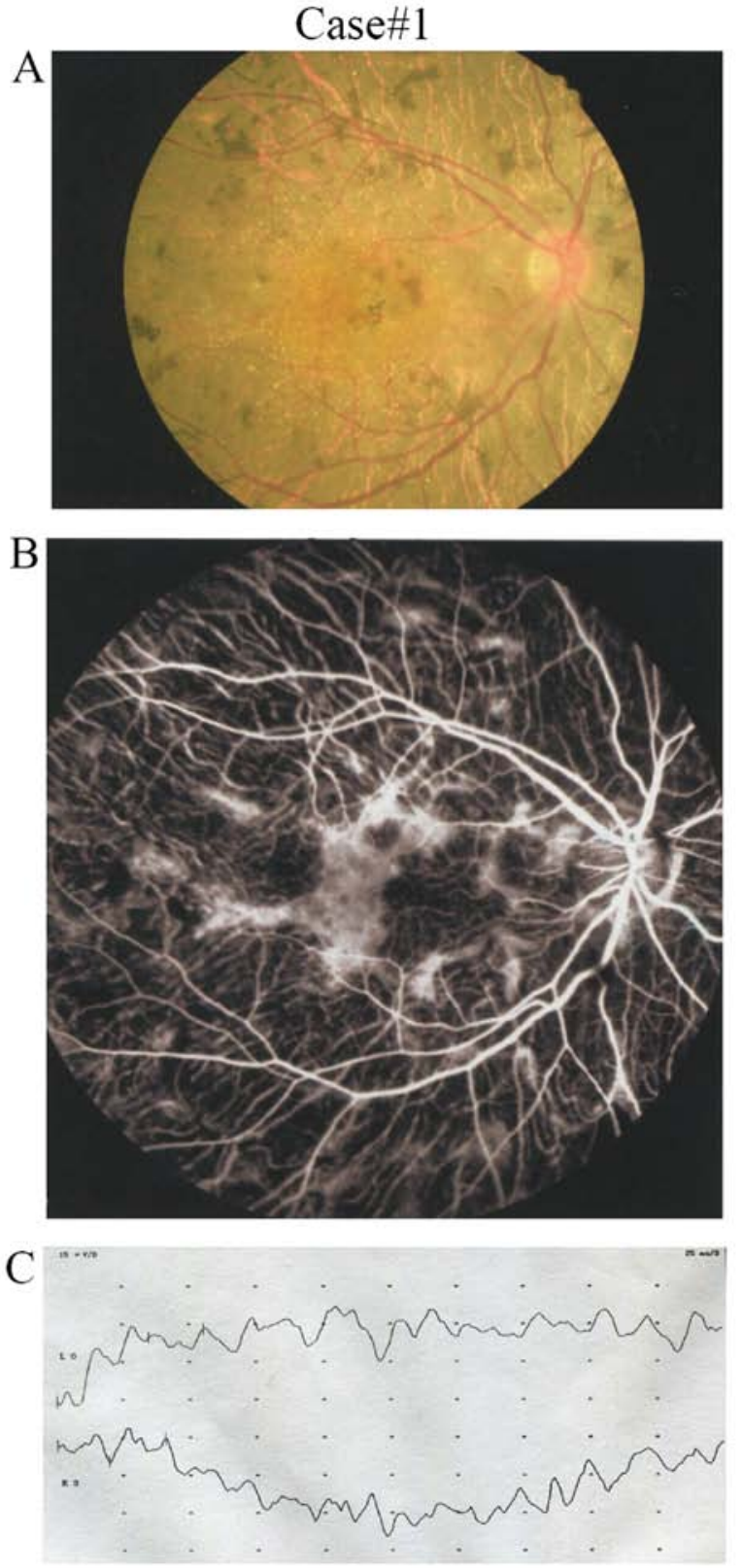
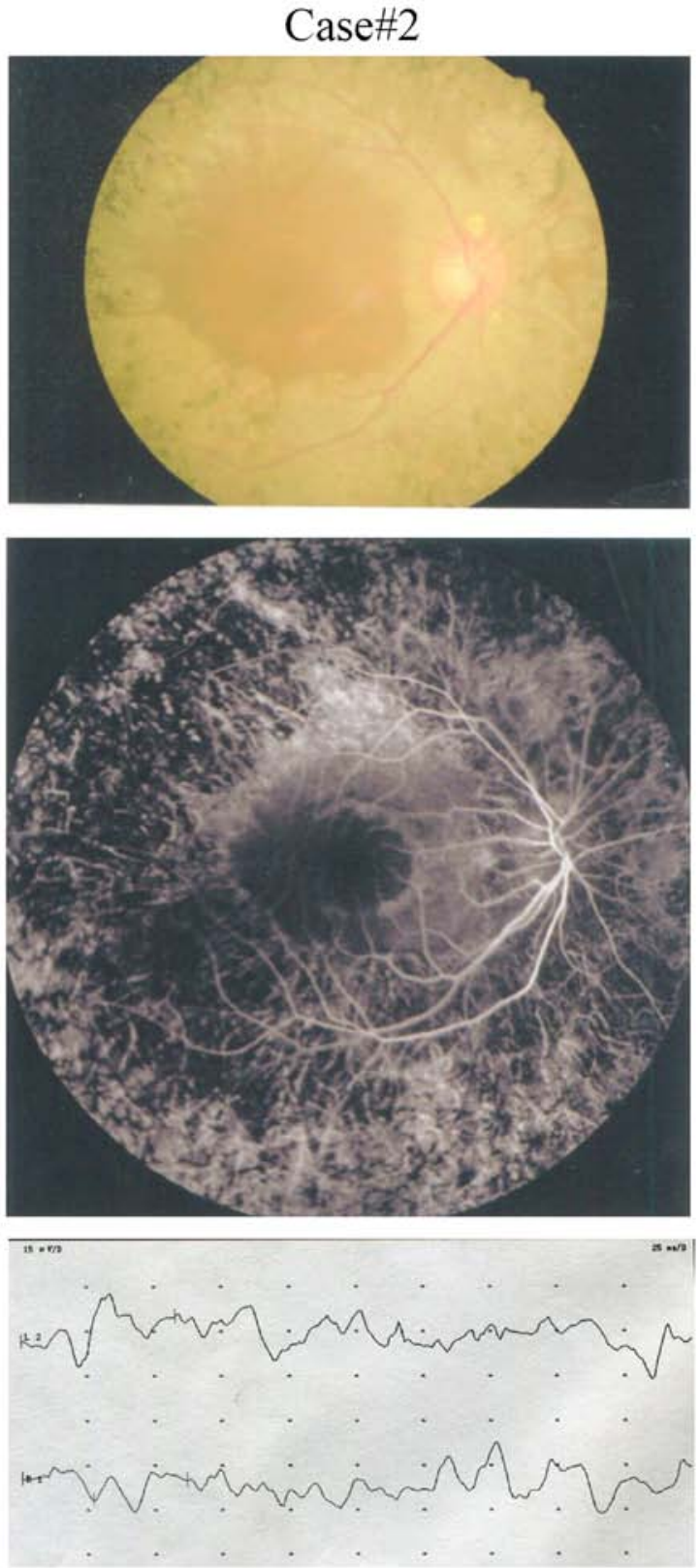

Figure 2. Representative results following ophthalmological assessment of the probands. (A) Fundus photographs of eyes (case\#1 and case\#2) showing peripheral pigmentation and retinal vessel attenuation. (B) Fluorescein angiography (FA) images showing that the hyperfluorescent flecks extend to the midperipheral retina and fluorescence blocking by the pigment mottling in the retina. (C) Electroretinograms showed no detectable rod and cone responses.

\section{Discussion}

In the present study, we have identified a novel homozygous frameshift mutation, c.1445dupT (p.F482fs), in the PROMI gene that appears to have caused autosomal recessive RP in two Chinese patients with sporadic RP. The mutation co-segregated with the disease as the two affected individuals were homozygous, whereas the parents of case\#1 and case\#2 carried only one mutation. The mutation was absent in the 200 normal controls. The affected individuals presented with classic RP disease and rapidly progressed to show severe visual impairment.

The PROM1 gene (MIM: 604365) is located at 4p15.32 and encodes a five-transmembrane (TM) domain glycoprotein with two short N (extracellular)- and C (cytoplasmic)-terminal tails, and two large N-glycosylated extracellular loops (between TM2 and -3 , and TM4 and -5). PROM1 has been identified as a hematopoietic, neuroepithelial and cancer stem cell marker (13-15). PROM1 expression is widespread throughout human tissues, including rod and cone photoreceptors. In the retina, $P R O M 1$ is found at the base of the photoreceptor outer segments (OSs). The function of PROM1 in the retina remains unknown; however, it has been demonstrated that mutations in PROM1 result in autosomal recessive RP, autosomal dominant macular degeneration and cone-rod dystrophy (16-23). To date, 35 different PROM1 mutations have been reported (Human Gene Mutation Database, http://www.hgmd.cf.ac.uk/). Notably, there is an association between the mutation type in PROM1 and the phenotype. Missense mutations are associated with autosomal dominant bull's eye maculopathy (20). 


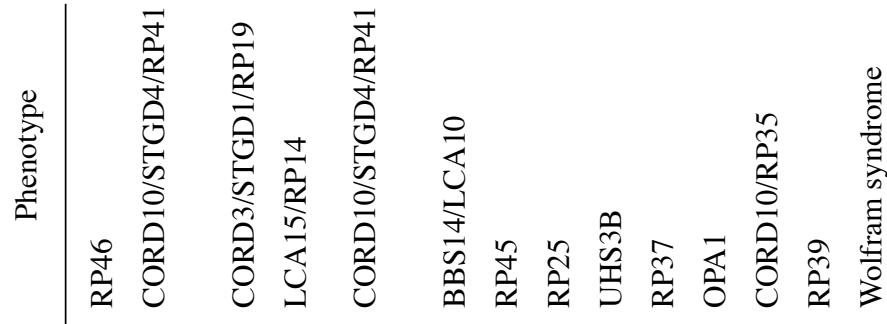

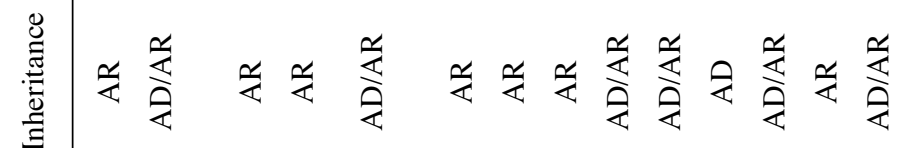

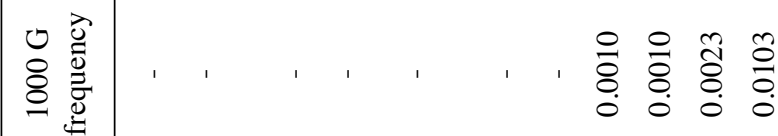

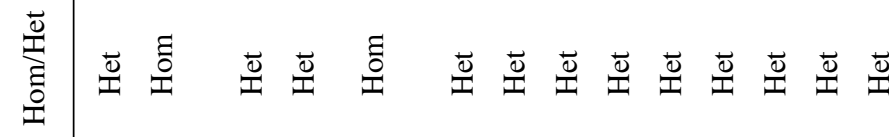

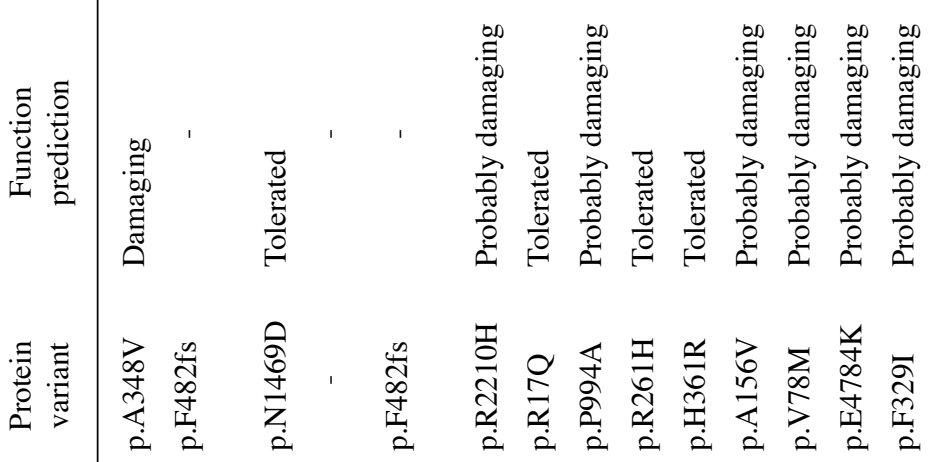

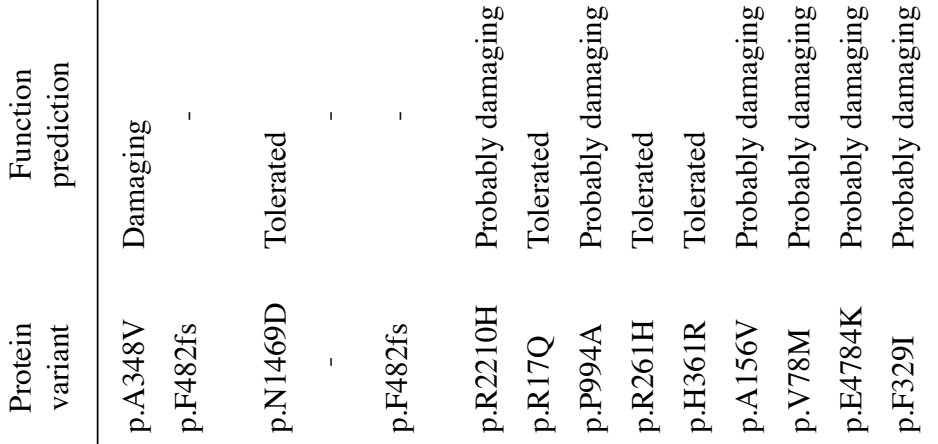

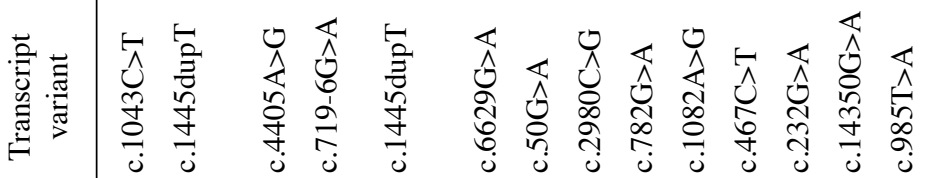

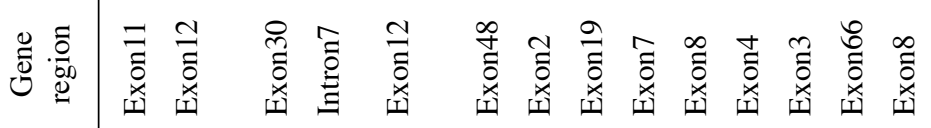

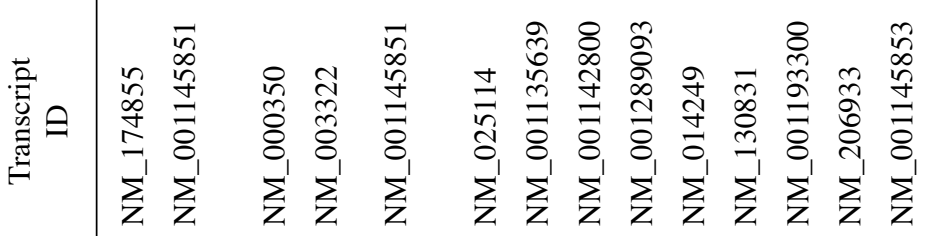

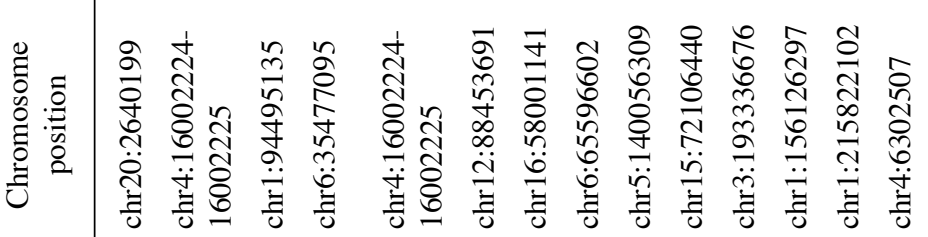

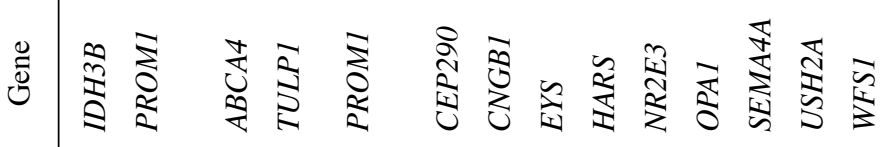




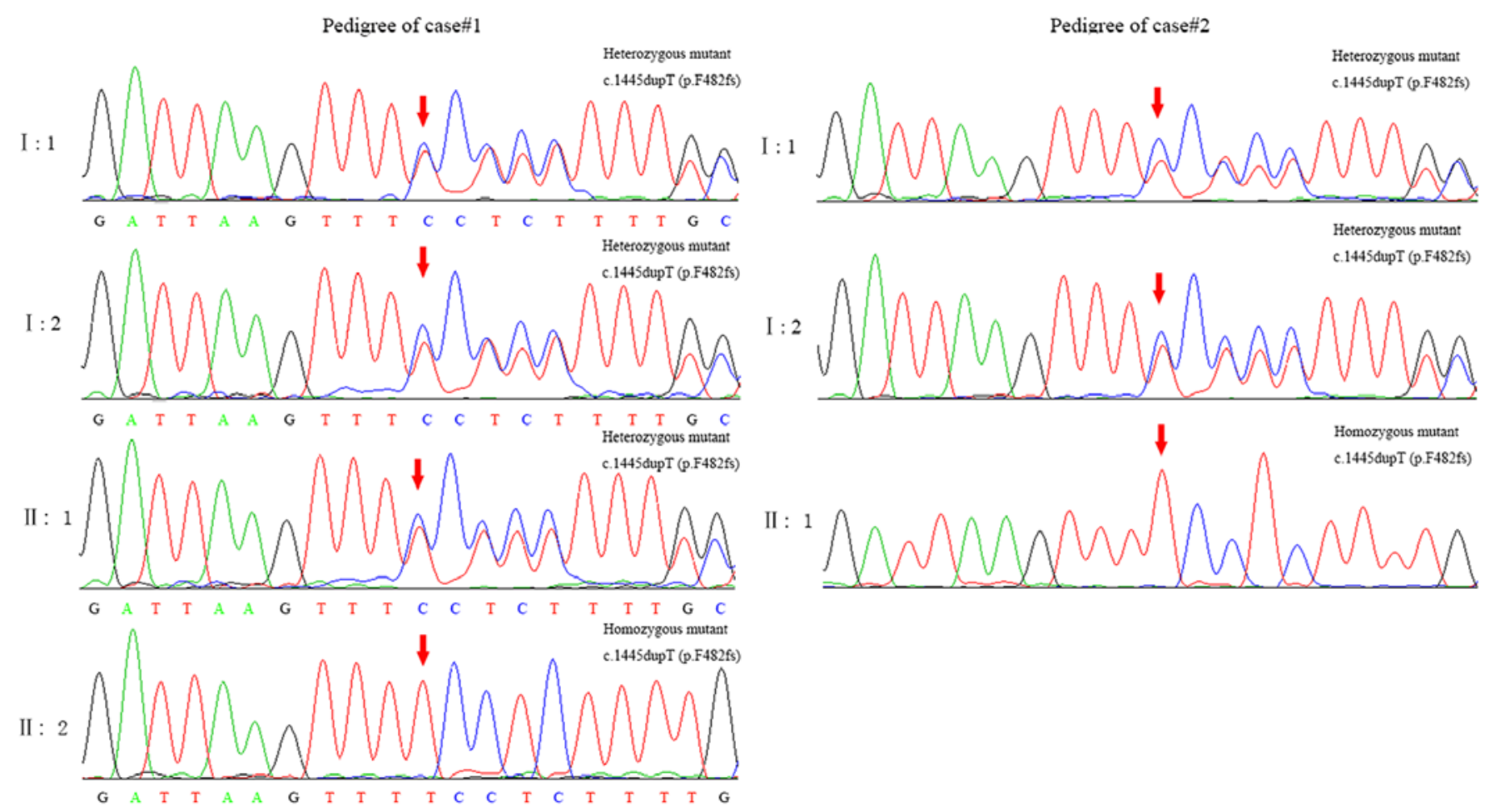

Figure 3. Identification of a mutation in prominin 1 (PROM1) gene. Sanger sequencing of case\#1 confirmed that the affected sibling (case\#1, II: 2) was homozygous for c.1445dupT (p.F482fs) whereas the parents (I: 1 and I:2) and the older brother (II: 1) of the proband were unaffected heterozygous carriers of c.1445dupT (p.F482fs), showing complete co-segregation of the mutation with the disease phenotype in this family. The c.1445dupT mutation was also observed in case\#2.

Mutation

p.Phe $482 \mathrm{fs}$

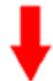

H. sapiens

CGV CGYDRHATPTTRGCVSNTGG F FXXXXXXXXXXXXXXXXXXXX

P. troglodytes CGVCGYDRHATPTTRGCVSNTGGVFLMVGVGLSFLFCWILMIIVVLTFVF

M. mulatta CGVCGYDRHATPTTRGCVSNTGGVFLMVGVGLSFLFCWILMIIVVLTFVF

C. lupus CGVCGYDRHATPTTRGCVSNTGGVFLMVGVGLSFLFCWILMIIVVLTFVF CGTFGYDRHATPTRRGCVSNTGGIFL M V G G ISFLFCWILMTIVVLTFVI

M. musculus

R. norvegicus C GVFGYDKHATPTRRGCVSNTGGIFLMAGVGFGFLFCWIL M ILVVLTFVV

G. gallus CGVFGYDKRATPTRRGCVSNTGGIFLMAGVGFSFLFCWILMILVVLTFVV

D. rerio C G T C G Y D HASPTTRGCISNTGGNFLMAGVGFSFLFSWVLMIVVVLTFVT CGILGFDRHASPTTRGCVSNTGGNFLMAGVGFSFFSWVLMGVITALFLA

Figure 4. Prominin 1 (PROM1) protein sequences from different species. Orthologous protein sequence alignment shows that the region is highly evolutionarily conserved in amino acid residues from position 482 in PROMI.

Nonsense and frameshift mutations have been associated with autosomal recessive RP and severe cone-rod dystrophy with macular degeneration and night blindness $(17,19,23)$.

In the present study, we have identified a novel homozygous frameshift mutation in the PROM1 gene that is responsible for severe RP in two patients with sporadic RP from China. Sanger sequencing of case\#1 confirmed that the unaffected sibling (II: 1) of the proband, her father (I: 1) and mother (I: 2) were found to carry the mutation c.1445dupT (p.F482fs) in a heterozygous state. The mutation shows complete co-segregation with the disease phenotype in this family. The same mutation was then found in an unrelated patient with RP (case\#2). It remains difficult to define a disease-causing mutation, particularly as there are no readily available functional assays to determine the phenotypic effects of specific variants. The potential pathogenicity of the filtered variants was then interpreted according to the existing and proposed ACMG guidelines (24). This frameshift mutation resulted in a completely different amino acid sequence from 483-amino acid residues of PROM1 gene. The 
frame-shift mutation occurred in half of the gene in a region that is highly conserved for PROM1, which was demonstrated by analyzing orthologs from 9 different species, namely Homo sapiens, Pan troglodytes, Macaca mulatta, Canis lupus, Mus musculus, Rattus norvegicus, Gallus gallus and Danio rerio. Such a high degree of conservation indicates the functional importance of the relevant amino acid sequence. This mutation was absent in the 200 normal controls and public databases including 1000 Genomes Project, Exome Variant Server for the NHLBI Exome Sequencing Project (http://evs.gs.washington.edu/EVS/), ExAC database (http://exac.broadinstitute.org/) and our in-house database generated from 1,977 WES samples, excluding them as common polymorphisms.

In conclusion, a homozygous mutation c.1445dupT (p.F482fs) in the PROM1 gene was identified in two Han Chinese subjects with RP by WES. The novel homozygous mutation in PROMI appears to be the cause of RP. The mutation further expands the existing spectrum of PROM1 mutations in patients with RP, thereby assisting in the molecular diagnosis of RP and enhancing our understanding of genotype-phenotype correlations in order to provide effective genetic counseling.

\section{Acknowledgements}

The present study was supported by the National Natural Science Foundation of China (grant no. 81271048) and the Foundation for Transfer of Scientific and Technological Achievements of Sichuan Province (grant no. 15010118).

\section{References}

1. Chizzolini M, Galan A, Milan E, Sebastiani A, Costagliola C and Parmeggiani F: Good epidemiologic practice in retinitis pigmentosa: from phenotyping to biobanking. Curr Genomics 12: 260-266, 2011.

2. Hartong DT, Berson EL and Dryja TP: Retinitis pigmentosa. Lancet 368: 1795-1809, 2006.

3. Ferrari S, Di Iorio E, Barbaro V, Ponzin D, Sorrentino FS and Parmeggiani F: Retinitis pigmentosa: genes and disease mechanisms. Curr Genomics 12: 238-249, 2011.

4. Schorderet DF and Escher P: NR2E3 mutations in enhanced S-cone sensitivity syndrome (ESCS), Goldmann-Favre syndrome (GFS), clumped pigmentary retinal degeneration (CPRD), and retinitis pigmentosa (RP). Hum Mutat 30 1475-1485, 2009.

5. Ku CS, Cooper DN, Polychronakos C, Naidoo N, Wu M and Soong R: Exome sequencing: dual role as a discovery and diagnostic tool. Ann Neurol 71: 5-14, 2012.

6. Ng SB, Buckingham KJ, Lee C, Bigham AW, Tabor HK, Dent KM, Huff CD, Shannon PT, Jabs EW, Nickerson DA, et al: Exome sequencing identifies the cause of a Mendelian disorder. Nat Genet 42: 30-35, 2010.

7. Worthey EA, Mayer AN, Syverson GD, Helbling D, Bonacci BB, Decker B, Serpe JM, Dasu T, Tschannen MR, Veith RL, et al Making a definitive diagnosis: successful clinical application of whole exome sequencing in a child with intractable inflammatory bowel disease. Genet Med 13: 255-262, 2011.

8. Yang Y, Muzny DM, Reid JG, Bainbridge MN, Willis A, Ward PA, Braxton A, Beuten J, Xia F, Niu Z, et al: Clinical whole-exome sequencing for the diagnosis of Mendelian disorders. N Engl J Med 369: 1502-1511, 2013.
9. Yang Y, Muzny DM, Xia F, Niu Z, Person R, Ding Y, Ward P, Braxton A, Wang M, Buhay C, et al: Molecular findings among patients referred for clinical whole-exome sequencing. JAMA 312: 1870-1879, 2014

10. Zhou Y, Tao S, Chen H, Huang L, Zhu X, Li Y, Wang Z, Lin H, Hao F, Yang Z, et al: Exome sequencing analysis identifies compound heterozygous mutation in ABCA4 in a Chinese family with Stargardt disease. PLoS One 9: e91962, 2014.

11. Gong B, Wei B, Huang L, Hao J, Li X, Yang Y, Zhou Y, Hao F, Cui Z, Zhang D, et al: Exome sequencing identified a recessive RDH12 mutation in a family with severe early-onset retinitis pigmentosa. J Ophthalmol 2015: 942740, 2015.

12. Richards CS, Bale S, Bellissimo DB, Das S, Grody WW, Hegde MR, Lyon E and Ward BE; Molecular Subcommittee of the ACMG Laboratory Quality Assurance Committee: ACMG recommendations for standards for interpretation and reporting of sequence variations: Revisions 2007. Genet Med 10: 294-300, 2008.

13. Bauer N, Fonseca AV,Florek M, Freund D, Jászai J, Bornhäuser M, Fargeas CA and Corbeil D: New insights into the cell biology of hematopoietic progenitors by studying prominin-1 (CD133). Cells Tissues Organs 188: 127-138, 2008.

14. Walker TL, Wierick A, Sykes AM, Waldau B, Corbeil D, Carmeliet $P$ and Kempermann G: Prominin-1 allows prospective isolation of neural stem cells from the adult murine hippocampus. J Neurosci 33: 3010-3024, 2013.

15. Akita M, Tanaka K, Matsumoto S, Komatsu K and Fujita K: Detection of the hematopoietic stem and progenitor cell marker CD133 during angiogenesis in three-dimensional collagen gel culture. Stem Cells Int 2013: 927403, 2013.

16. Zhang Q, Zulfiqar F, Xiao X, Riazuddin SA, Ahmad Z, Caruso R, MacDonald I, Sieving P, Riazuddin S and Hejtmancik JF: Severe retinitis pigmentosa mapped to $4 \mathrm{p} 15$ and associated with a novel mutation in the PROM1 gene. Hum Genet 122: 293-299, 2007.

17. Maw MA, Corbeil D, Koch J, Hellwig A, Wilson-Wheeler JC, Bridges RJ, Kumaramanickavel G, John S, Nancarrow D, Röper K, et al: A frameshift mutation in prominin (mouse)-like 1 causes human retinal degeneration. Hum Mol Genet 9: 27-34, 2000 .

18. Yang Z, Chen Y, Lillo C, Chien J, Yu Z, Michaelides M, Klein M, Howes KA, Li Y, Kaminoh Y, et al: Mutant prominin 1 found in patients with macular degeneration disrupts photoreceptor disk morphogenesis in mice. J Clin Invest 118: 2908-2916, 2008.

19. Permanyer J, Navarro R, Friedman J,Pomares E,Castro-Navarro J, Marfany G, Swaroop A and Gonzàlez-Duarte R: Autosomal recessive retinitis pigmentosa with early macular affectation caused by premature truncation in PROM1. Invest Ophthalmol Vis Sci 51: 2656-2663, 2010

20. Michaelides M, Gaillard MC, Escher P, Tiab L, Bedell M, Borruat FX, Barthelmes D, Carmona R, Zhang K, White E, et al: The PROM1 mutation p.R373C causes an autosomal dominant bull's eye maculopathy associated with rod, rod-cone, and macular dystrophy. Invest Ophthalmol Vis Sci 51: 4771-4780, 2010.

21. Mayer AK, Rohrschneider K, Strom TM, Glöckle N, Kohl S, Wissinger B and Weisschuh N: Homozygosity mapping and whole-genome sequencing reveals a deep intronic PROM1 mutation causing cone-rod dystrophy by pseudoexon activation. Eur J Hum Genet 24: 459-462, 2016.

22. Khan AO and Bolz HJ: Pediatric cone-rod dystrophy with high myopia and nystagmus suggests recessive PROM1 mutations. Ophthalmic Genet 36: 349-352, 2015.

23. Pras E, Abu A, Rotenstreich Y, Avni I, Reish O, Morad Y, Reznik-Wolf $\mathrm{H}$ and Pras E: Cone-rod dystrophy and a frameshift mutation in the PROM1 gene. Mol Vis 15: 1709-1716, 2009.

24. Richards S, Aziz N, Bale S, Bick D, Das S, Gastier-Foster J, Grody WW, Hegde M, Lyon E, Spector E, et al; ACMG Laboratory Quality Assurance Committee: Standards and guidelines for the interpretation of sequence variants: A joint consensus recommendation of the American College of Medical Genetics and Genomics and the Association for Molecular Pathology. Genet Med 17: 405-424, 2015. 